\title{
Comunidades terapéuticas. Situación actual y perspectivas de futuro
}

\author{
Llorente del Pozo, J.M.* Fernández Gómez C.** \\ ${ }^{*}$ Responsable del C.T.M.E. Foronda.Osakidetza - Servicio Vasco de Salud -Vitoria \\ * Investigador colaborador del C.T.M.E. Osakidetza - Servicio Vasco de Salud -Vitoria
}

Enviar correspondencia a:

Dr. Juan M. Llorente del Pozo. C.T.M.E. - Foronda (Osakidetza). 01196 Foronda - Alava (Spain). Tlfn. 0034945 265282. Fax 0034945 272048. E. Mail: jllorente@correo.cop.es

\section{Resumen}

Las Comunidades Terapéuticas Profesionales en España, ofrecen un modelo de rehabilitación muy útil para un sector de drogodependientes que necesitan un entorno seguro y una intervención más intensiva. Estos programas se están adaptando a las nuevas características de los usuarios.

Actualmente un porcentaje cada vez mayor de adictos presenta trastornos psiquiátricos concurrentes o pasados, especialmente trastornos afectivos y de personalidad. Los datos muestran que los usuarios de C.T. presentan en muchos casos una larga carrera adictiva y antecedentes de múltiples tratamientos seguidos de recaídas. Cronicidad y comorbilidad aparecen habitualmente asociadas a peores resultados en los tratamientos. Las CC.TT. profesionalizadas existentes en España podrían beneficiarse de nuevos tratamientos que están demostrando utilidad en pacientes crónicos (tratamiento con metadona), y en pacientes con trastornos psiquiátricos (medicación psicofarmacológica). Existen algunas experiencias en USA y otros países Europeos que ilustran la adaptación de CC.TT. para pacientes en mantenimiento con metadona y para pacientes con doble diagnóstico.

Palabras clave: Comunidad Terapéutica; adictos a drogas; rehabilitación, metadona, psicofármacos

\section{Summary}

The Professional Therapeutic Communities in Spain offer a very useful rehabilitation scheme for a drug dependent sector needs a safe environment and a more intensive intervention. These programs are adapted to the new characteristics of the users.

At present, an ever increasing percentage of addicts display current, or past, psychiatric problems, especially affective or personality problems. The data show that in many cases the T.C users show a long history of addiction and have records of multiple treatment followed by relapses. Chronicity and co-morbidity usually appear to be associated with the worst results within the treatment. The existing professional T.C's in Spain may be able to benefit from new treatments which are proving themselves useful to chronic patients (methadone treatment) and to patients with psychiatric problems (psycho-pharmacological treatment). There is some experience in the USA and in other European countries which shows how the T.C's can be adapted to patients being kept on methadone and for patients with dual diagnosis.

Key words: Therapeutic Community, Drug abusers, rehabilitation, methadone, psycho- pharmacological treatment

\section{II - INTRODUCCION}

I término comunidad terapéutica se acuñó en hospitales psiquiátricos del Reino Unido en la década de los 50 (1). Una década más tarde, el término comunidades terapéuticas comienza a utilizarse para denominar determinados tratamientos residenciales libres de drogas para sujetos dependientes de sustancias, aunque estos programas surgieron independientemente del contexto del hospital psiquiátrico. La comunidad terapéutica (C.T.) como tratamiento para el abuso y dependencia de drogas surgió por los años 60 en Norteamérica, como una alternativa a los tratamientos convencionales. El concepto base de este modelo era la autoayuda, y fue desarrollado inicialmente por alcohólicos en recuperación y drogodependientes. Así, aunque Alcohólicos Anónimos y Synanon son sus antecedentes más reconocidos, el modelo de comunidad terapéutica es más antiguo y ha sido aplicado en diversos campos de asistencia y apoyo comunitario.

El modelo de las comunidades terapéuticas para toxicómanos engloba una gran variedad de programas heterogéneos en cuanto a origen y fundamentos, 
objetivos y métodos de intervención. El estudio del origen y desarrollo histórico de las comunidades terapéuticas excede el objetivo de esta ponencia, a este fin puede consultarse otros trabajos $(2,3,4)$. Actualmente existe una variedad considerable de modelos de intervención que se autodenominan en ocasiones "comunidades terapéuticas"; algunas de las cuales se basan en ideologías pseudo - científicas o religiosas. El marco teórico global de comunidad terapéutica tiene un referente en un modelo Americano, (más estructurado y normativo, con bases teóricas próximas a la modificación de conducta y al movimiento de autoayuda) y un referente Europeo (basado en la psiquiatría comunitaria y el aprendizaje social (5).

El término de CC.TT. profesionales en España surgió en la literatura en el año 1985 (2), para definir algunos centros de internamiento para toxicómanos con una finalidad de reinserción social, y distinguirlos de centros que se configuraban como meros lugares de exclusión y control. Estas CC.TT. profesionales se asociaron como colectivo en el año 1986 en la Asociación de Profesionales de Comunidades Terapéuticas para Toxicómanos (APCTT) y recientemente en la Asociación de Intervinientes en Comunidad Terapéutica (I.C.T.). Estos colectivos han definido unas bases comunes que articulan su modelo de intervención (6) y un sistema de evaluación de su actividad (7) que sigue siendo objeto de debate y mejora. En el último informe cuadrienal se expone la actividad de 22 CC.TT. profesionales, donde desempeñan su actividad en torno a 260 profesionales contratados, con un volumen anual de atendidos cercano a los 2.100 usuarios (7). El marco teórico de estos programas intenta alcanzar un equilibrio entre los dos referentes teóricos de las CC.TT, entre la modificación conductual, la organización de la autonomía y competencias individuales de los usuarios, las aportaciones del movimiento de auto-ayuda y la psicoterapia.

La red Proyecto Hombre está formada por programas que comenzaron a funcionar en España aproximadamente en 1984-1986, año en que se fundó la Confederación de programas proyecto Hombre en España. Su programa está influido por el Centro Italiano de Solidaridad y de sus referencias Americanas (Daytop Village y otros programas semejantes). Al igual que éstos, el programa de proyecto Hombre se basa en el concepto de auto-ayuda, el grupo, y un complejo sistema de actividades educativas, de ocupación del tiempo y organización que constituye un programa educativo-terapéutico global. Aunque los programas de esta red son más que una comunidad terapéutica, su modelo de tratamiento está fuertemente influido por las CC.TT. Americanas y está bien consolidado en nuestro país.

En la actualidad el modelo de C.T. está extendido y consolidado en nuestro entorno, realizando una contribución sustancial en el campo de la asistencia en dro- godependencias. A título de ejemplo, el catálogo de centros de la Delegación del Gobierno para el Plan Nacional Sobre Drogas incluye un número aproximado de 104 programas denominados comunidades terapéuticas, entre un listado de centros públicos o privados con financiación pública de asistencia a drogodependientes durante el año 1997 (8). Este número de centros, que no incluye todos los programas que en España se autocalifican a si mismos como CC.TT.; atendieron durante ese mismo año a un número aproximado de 7.000 drogodependientes (principalmente dependientes a heroína (9).

Las comunidades terapéuticas (CC.TT.) contemporáneas han desarrollado y evolucionado muy significativamente respecto a sus orígenes históricos. Actualmente el término comunidad terapéutica resulta genérico y poco preciso, aplicándose a una gran variedad de instituciones residenciales, de titularidad pública o privada, que han ido alcanzando en algunos casos un alto grado de sofisticación. Un número importante de ellas mantiene como referente el modelo de comunidad terapéutica tradicional Americano, mientras otras CC.TT. más profesionalizadas han adaptado este modelo, combinándolo con postulados de la atención en salud mental y servicios asistenciales comunitarios. La C.T. tradicional, cercana al modelo Americano, suele ofertar dentro de un mismo programa una variedad de servicios de modo coordinado: consejo vocacional, terapia ocupacional, actividades recreativas, terapia individual y de grupo, y servicios educativos y a las familias, asistencia y asesoramiento médico, legal e información y acceso a servicios comunitarios. Las CC.TT. Españolas privadas o públicas suelen estar integradas en redes asistenciales más amplias o bien utilizan otros servicios sociales y comunitarios para satisfacer necesidades específicas de sus usuarios.

En la C.T. tradicional, el "terapeuta principal", agente terapéutico y modelo a seguir es la propia comunidad, formada por los pares y el staff como modelos de rol que modelan un cambio personal exitoso. En el modelo Europeo, junto con los componentes grupales y comunitarios, los agentes activos del cambio suelen ser estrategias formales de intervención, basadas en las ciencias médicas y sociales. En ambos casos, sin embargo, la interacción diaria, continuada e intensiva constituye un elemento esencial del modelo de intervención. Finalmente, el modelo tradicional de C.T. en la rehabilitación del abuso de drogas se basa en una perspectiva explícita sobre el trastorno adictivo, el cliente o paciente, el proceso de recuperación y el modo de vida correcto y saludable. Esta perspectiva moldea la estructura de la organización, el personal y el proceso de tratamiento. Existen diferencias sustanciales en la visión y perspectiva que caracteriza a las CC.TT. Españolas. Las CC.TT. integradas en la Red de Proyecto Hombre podrían considerarse como más 
cercanas al modelo tradicional Americano, mientras que otros programas que han venido a denominarse CC.TT. profesionales estarían más cerca de un modelo Europeo y de otras CC.TT. "modificadas" que han ido surgiendo en USA durante los últimos años.

\section{III - LA COMUNIDAD COMO AGENTETERAPÉUTICO}

A diferencia de otras modalidades de tratamiento, la C.T. constituye un "medio terapéutico", cuyo régimen diario consiste en actividades estructuradas y no estructuradas e interacciones sociales que se desarrollan en situaciones y entornos formales e informales, y que constituyen entre todas las intervenciones terapéuticas durante el proceso.

Un día típico en una C.T. implica un periodo muy estructurado, que se inicia aproximadamente a las 7.00 a.m. y finaliza aproximadamente a las 23.00 p.m. Durante este tiempo los usuarios participan en una variedad de reuniones, actividades ocupacionales, grupos de encuentro y otros grupos terapéuticos, consejo y terapia individual, y actividades recreativas. La interacción entre estas actividades educativas, terapéuticas, y sociales constituye la esencia de la comunidad como agente terapéutico.

La C.T. se beneficia del papel de los pares como agente activo en el cambio del usuario, a través del fortalecimiento de los vínculos interpersonales. Del mismo modo que el papel de los pares es ampliamente reconocido en la iniciación, desarrollo y mantenimiento de los trastornos adictivos, la C.T. se basa también en la utilidad del grupo de iguales en el cambio de la conducta adictiva y otras conductas relacionadas. Los compañeros son una fuente incomparable de feedback de evaluación y apoyo, reforzamiento para las conductas positivas, instrucción, modelamiento y sugerencias para el cambio. La C.T. proporciona un espacio único para que estas interacciones sean intensivas, continuadas, programadas y desarrolladas de modo estructurado, lo que facilita la satisfacción de necesidades relacionales en el usuario y a la vez permiten la estructuración de las relaciones de este consigo mismo y con los demás, preparándole para la integración posterior en otros núcleos familiares y sociales. Así, el aprendizaje observacional o vicario, el control de las contingencias de la conducta bajo control del equipo, la ayuda mutua y las estrategias grupales para facilitar el cambio motivacional, actitudinal y comportamental constituyen un elemento básico del modelo de C.T. Estas actividades comunitarias se desarrollan a través de reuniones diarias, seminarios frecuentes, sesiones de terapia grupal y la interacción permanente en el marco de la C.T. Durante las tareas ocupacionales, grupos, reuniones, actividades recreativas y tiempo personal y de interacción social, los residentes se transmiten entre si los mensajes que constituyen el espíritu y esencia de la comunidad. Así, la importancia de la autoayuda es evidente, por la amplia variedad de tareas que son responsabilidad de los residentes: a menudo todas las tareas de cocina, limpieza, mantenimiento básico de la C.T. son responsabilidad de los residentes, incluso en ocasiones éstos conducen reuniones y grupos de encuentro.

\section{IV - ELEMENTOS TERAPÉUTICOS FORMALES DE LA C.T.}

Las CC.TT. profesionalizadas incorporan a su programa terapéutico estrategias formales de intervención muy variadas. Las estrategias terapéuticas formales aplicadas van desde el tratamiento psiquiátrico hasta la aplicación de técnicas de tratamiento psicológico de índole diversa, según la orientación teórica de la C.T. (terapia cognitivo-conductual, familiar - sistémica, de orientación psicoanalítica, psicodrama, Gestalt, psicoterapia centrada en el cliente, etc.).

En el CTME de Foronda el tratamiento desarrollado para la deshabituación de sustancias se basa fundamentalmente en las teorías cognitivo - conductuales, el aprendizaje social y las teorías motivacionales sobre el cambio en las conductas adictivas. Además de diversos componentes típicos de las comunidades terapéuticas, el CTME aplica estrategias conductuales orientadas a la modificación de conducta, estrategias cognitivo - conductuales, técnicas de modificación cognitiva y técnicas motivacionales. Las técnicas conductuales se basan en el condicionamiento operante e implican la aplicación sistemática de contingencias para determinadas conductas que favorecen o dificultan la deshabituación. Aunque la C.T. no está estructurada formalmente como una unidad de modificación de conducta, constituye una oportunidad para la observación conductual y la aplicación de técnicas basadas en el reforzamiento diferencial de respuestas. Las estrategias cognitivo - conductuales van orientadas a la adquisición de habilidades diversas (habilidades de afrontamiento del estrés y de los deseos de consumo, autocontrol, habilidades sociales, prevención de recaídas, etc.), y a la preparación del sujeto para el estilo de vida abstinente. Solamente se subraya que estas estrategias cognitivo - conductuales se enfocan en ayudar al paciente a identificar y modificar las cadenas de comportamientos y pensamientos que conducen directa o indirectamente al uso de drogas. Estas técnicas incluye: a) el aprendizaje y práctica de habilidades para el afrontamiento del craving agudo y crónico y otras situaciones de riesgo para el uso de drogas; b) promoción y reforzamiento de habilidades sociales y conductas compatibles con el estilo de vida abstinente e incompatibles con el uso 
de drogas; y c) técnicas de modificación cognitiva. Las técnicas cognitivas se basan en la adaptación de las teorías cognitivas de Beck (10) y las teorías del aprendizaje social (11), y se enfocan en la identificación de patrones de pensamiento desadaptativos como instrumento para modificar o eliminar estados afectivos negativos y conductas desadaptativas. Las técnicas motivacionales aplicadas en el CTME de Foronda consisten en un acercamiento empático y escucha reflexiva, orientada a esclarecer y aumentar la motivación del paciente hacia el cambio, mediante el diálogo acerca de los pros y contra de una conducta específica, la exploración de las metas y objetivos del paciente y la ambivalencia acerca del logro de esas metas. En las CC.TT., junto con las estrategias de intervención individuales, las técnicas grupales constituyen la base de la intervención psicológica. Las técnicas específicas utilizadas varían significativamente, según la orientación teórica del programa: técnicas modificadas del psicoanálisis, terapia interpersonal, racional emotiva, Gestalt, Psicodrama, etc. El contexto grupal puede constituirse como un marco de apoyo, terapéutico o educativo. Según la tolerancia de los pacientes para las dinámicas de interacción en el grupo, se utilizan técnicas de confrontación de la negación, y de las discrepancias entre actitudes e intenciones manifiestas y la conducta observable, el manejo de conflictos interpersonales y relaciones cercanas, la comunicación de afectos y experiencias traumáticas, etc. En general, las técnicas de intervención grupal en la C.T. suministran una oportunidad privilegiada para la identificación con otros individuos que se enfrentan y resuelven dificultades semejantes. En la práctica, las técnicas de grupo ayudan a identificar y comprender el impacto que el abuso y dependencia de drogas en las vidas de cada uno de los componentes del grupo, a aprender acerca de los sentimientos y reacciones propios y ajenos y a comunicar y escuchar necesidades y sentimientos de un modo más adaptativo.

Dentro de las estrategias grupales, se utilizan los grupos de encuentro para confrontar actitudes y comportamientos negativos y reforzar cambios positivos en tales actitudes y comportamiento. En las CC.TT. más tradicionales, estos grupos de encuentro constituyen la piedra angular de los procesos grupales en la C.T. Son especialmente importantes los grupos didácticos tutoriales, orientados a enseñar y practicar habilidades interpersonales y conceptos relacionados con la recuperación. En algunas CC.TT. especialmente en las más cercanas al modelo tradicional Americano tienen una gran importancia los grupos orientados a descubrir material sensitivo y biográfico (grupos sonda, etc.). Estos grupos proporcionan al equipo información clínica muy valiosa para comprender los antecedentes históricos del residente. Esta información obtenida en los grupos sonda es crítica para la planificación del tratamiento, así como para aumentar el sentimiento de cercanía e identificación, apoyo, comprensión y empatía. Los grupos intensivos (maratón) son sesiones de larga duración (hasta 18-36 horas) que tienen por objetivo iniciar la resolución de experiencias vitales que han podido dificultar el desarrollo personal de los usuarios. Otros grupos específicos orientados a temas de pareja, familia, etc. Son aplicados ad-hoc también habitualmente en las CC.TT., según la conveniencia y necesidad.

Además de las técnicas terapéuticas formales, la interacción usuario - equipo clínico proporciona un marco informal para el consejo, que supone una aportación significativa añadida a las sesiones de consejo individual. El consejo tiene a menudo una naturaleza didáctica y en ocasiones incluye el compartir experiencias personales y sentimientos entre consejero y usuario.

La asistencia sanitaria integral a los residentes constituye un elemento esencial de las CC.TT. Los usuarios de estos programas habitualmente presentan problemáticas orgánicas severas asociadas al abuso de drogas de larga evolución: hepatitis, tuberculosis, SIDA, etc. Estos trastornos requieren una supervisión regular acerca de su evolución, así como un cumplimiento estricto de los protocolos farmacológicos. Las CC.TT. profesionalizadas proporcionan una oportunidad óptima para alcanzar una adecuada supervisión, tratamiento y seguimiento de estas patologías orgánicas. Adicionalmente, los usuarios de las CC.TT. para drogodependientes frecuentemente presentan comorbilidad psiquiátrica con indicación de terapia farmacológica de apoyo (trastornos afectivos, psicóticos, de personalidad límite, etc.). Del mismo modo que en las patologías orgánicas señaladas, estas pautas de medicación psiquiátrica exigen una monitorización y supervisión para evitar el uso inadecuado o abuso. Este control puede efectuarse en la C.T. con mayor facilidad que en otros programas asistenciales no residenciales.

El consejo o terapia familiar es generalmente un elemento esencial del tratamiento en C.T. En muchas ocasiones los recursos familiares son integrados en el plan de tratamiento como agente terapéutico, o como instrumento de monitorización y apoyo a la conducta del usuario. En muchos casos, los pacientes tratados en las CC.TT. provienen de familias que se caracterizan por una comunicación deteriorada entre sus miembros; y por una dificultad para establecer límites y normas de comportamiento. Las intervenciones familiares específicas son aplicadas habitualmente cuando la abstinencia del paciente perturba de algún modo un estilo de vida familiar previamente bien establecido (aunque desadaptativo), o cuando otros miembros de la familia necesitan ayuda para adaptarse a un nuevo sistema de normas, objetivos, actitudes y comportamientos $(12,13)$. Las técnicas de intervención familiar pueden ir orientadas a las interacciones inter- 
personales y familiares que conllevan a un conflicto, o fortalecer comportamientos que ayuden a evitar las recaídas en pacientes con alto grado de implicación familiar. Las estrategias de intervención aplicadas en C.T. varían también según la orientación del programa, incluyendo técnicas de consejo, grupos multifamiliares, o terapia conductual, estratégica, estructural, dinámica o sistémica. Estas intervenciones incluyen las dirigidas al núcleo familiar del paciente, terapia de pareja, tratamiento concurrente para diferentes miembros de la familia, etc. Las metas más comunes de las intervenciones familiares son fortalecer el apoyo familiar hacia la abstinencia del paciente, suministrar información acerca de la evolución del paciente durante el tratamiento y acerca de sus actitudes hacia el consumo y abstinencia de drogas, favorecer el cumplimiento de las pautas del tratamiento y monitorizar la conducta del paciente en sus relaciones interpersonales y familiares, ocupacionales abstinencia y exposición a situaciones de riesgo.

Estas estrategias formales se complementan con una actitud de los usuarios como modelos de rol. De manera importante, la fuerza de la C.T. como contexto para el aprendizaje social se basa en la variedad y cualidad de sus modelos de rol. Se espera que todos los miembros de la comunidad sean modelos de rol para los demás. Uno de los atributos de estos modelos se caracteriza por un "act as if = actuar como si", consistente en que los residentes se comporten como las personas que deben ser, en lugar de cómo las personas que han sido, a pesar de las resistencias, percepciones y sentimientos contrarios. De este modo incorporan a la vida diaria el nuevo estilo actitudinal y conductual, maximizando las propias potencialidades. Este "act as if" no es simplemente un ejercicio de conformidad, sino más bien un mecanismo esencial para un cambio psicológico más completo, de modo que los cambios en sentimientos, "insight" y autopercepciones distorsionadas a menudo siguen al cambio conductual, en lugar de precederlo. Otro atributo esperado es "mostrar un interés responsable" hacia los demás, que consiste en ser consciente de la apariencia, actitudes, humor y rendimiento de los pares, confrontando los signos negativos. Este atributo tiene un significado semejante al concepto de ser "custodio" de los compañeros en la C.T. Finalmente, la autoayuda está omnipresente en la C.T., y es reforzada mediante la alabanza, confrontando las conductas y actitudes contrarias a esta implicación activa.

La supervisión o vigilancia del funcionamiento de la C.T. constituye un elemento esencial, en cuanto que sirve para verificar el cumplimiento de las premisas de la C.T. Típicamente en las CC.TT. más tradicionales, durante una o varias veces al día, componentes del equipo y usuarios con más experiencia revisan la C.T., examinando su condición y estado general, atendiendo a diversos aspectos: limpieza, cumplimiento de las rutinas, procedimientos de seguridad, moral y "tono psicológico" de los residentes. Muchas CC.TT. (más frecuentemente las que se distinguen del modelo tradicional americano) realizan procedimientos no programados y aleatorios de detección del uso de sustancias mediante técnicas analíticas de orina u otras. Estos procedimientos de supervisión aparentemente simple conllevan importantes implicaciones clínicas y objetivos para la gestión de la C.T. La aceptación voluntaria de una vulneración de la norma (uso de drogas u otra) implica la iniciación de una experiencia educativa, incluyendo la exploración e identificación de los antecedentes de tal conducta. La negación de tal conducta de uso de drogas no permitidas puede conducir a la terminación del tratamiento o al abandono prematuro.

\section{V - LA ADAPTACIÓN DE LAS COMUNIDADES TERAPEUTICAS EN ELTRATAMIENTO DEL ABUSO $Y$ DEPENDENCIA DE SUSTANCIAS}

Desde su origen, las Asociaciones formadas por las CC.TT. profesionales Españolas han buscado el intercambio permanente de experiencias y formación con otros profesionales e instituciones semejantes. Estos intercambios se están desarrollando actualmente también en el ámbito Europeo, dentro de las actividades programadas por T3E y ERIT (Federación Europea de Asociaciones de Intervinientes en Toxicomanías). En los últimos dos años, desde ERIT y con el apoyo de la Comisión Europea, las C.C.TT. profesionales Españolas representadas por ICT (Asociación de Intervinientes en Comunidad Terapéutica) están coordinando y jugando un papel principal en un grupo de trabajo Europeo para el estudio, desarrollo y aplicación de una normativa de calidad en comunidad terapéutica. Este grupo está formado por expertos en C.T. de varios países Europeos y sintoniza con las aspiraciones originales de las Asociaciones Españolas: la discusión, debate, estudio y desarrollo de los criterios propios de una C.T. profesional, como modelo de intervención orientado a la reinserción social de drogodependientes, integrado en una variedad de intervenciones posibles. Hasta la fecha, el grupo de trabajo ha desarrollado una serie de actividades, cuyos resultados más importantes son:

Un listado de Indicadores de calidad (con el asesoramiento técnico de Cesáreo Fernández Gómez) que incluye 120 indicadores y normas de calidad en una C.T., articulados en las siguientes áreas:

ASPECTOS FORMALES: Incluye 3 ítems que se refieren al cumplimiento de las normativas provinciales, regionales y nacionales sobre el funcionamiento de estos programas; así como a la importancia de la C.T. como recurso asistencial. 
MEDIOS FÍSICOS, ENTORNO, CONTROL SANITARIO: Incluye 14 ítems referidos a los medios físicos de la C.T., el entorno y clima social y el control sanitario en el programa.

ACTIVIDAD ECONÓMICA Y CUESTIONES FINANCIERAS: Agrupa 6 ítems referidos a los medios económicos de la C.T. y al control financiero.

EQUIPOTÉCNICO: COMPOSICIÓN, FORMACIÓN Y SUPERVISIÓN: Especifica 10 normas de calidad referidas a los recursos humanos de la C.T., en cuanto a su estructura, formación y gestión.

PROCEDIMIENTO DE ADMISIÓN: Incluye 9 ítems referidos a los procedimiento que la C.T. aplica durante el procedimiento de admisión de pacientes.

REGISTRO INDIVIDUAL DE PACIENTES: Agrupa 7 ítems referidos a la información que se registra sobre cada paciente admitido en la C.T.

DIAGNÓSTICO Y EVALUACIÓN DE PACIENTES: Son 12 ítems referidos a los procedimientos utilizados para valorar las necesidades de los pacientes antes, durante y al final del tratamiento.

TRATAMIENTO: GENERALIDADES, ACTIVIDADES Y PROGRAMAS EDUCATIVOS: Incluye 16 ítems referidos a las características generales del programa terapéutico y actividades.

FINALIZACIÓN, DERIVACIÓN Y SEGUIMIENTO: Incluye 7 ítems sobre el procedimiento seguido para la finalización del tratamiento y seguimiento posterior de pacientes.

DERECHOS DEL PACIENTE: Estos 8 ítems se refieren a los métodos seguidos por la C.T. en defensa de los derechos de los pacientes.

RELACIÓN CON LA COMUNIDAD Y CON OTROS SERVICIOS: Incluye 7 ítems referidos a la coordinación con otros programas asistenciales y con la comunidad.

GARANTÍA DE CALIDAD: Incluye 20 ítems referidos a las medidas que la C.T. aplica para garantizar la calidad en su asistencia a los pacientes.

Una Declaración Común Europea, a modo de consenso sobre algunos indicadores de calidad considerados como básicos desde varias CC.TT. profesionales Europeas.

Un Proyecto de trabajo Europeo, orientado a la investigación y desarrollo de la calidad en un modelo de comunidad terapéutica Europea.

En resumen, el listado es un instrumento que introduce un debate y consenso Europeo sobre los objetivos de calidad en comunidad terapéutica planteados desde los programas asociados en I.C.T. y otros afines. Estos temas de debate y desarrollo podrían resumirse en las siguientes grandes áreas: a) adaptación de los programas a las necesidades del usuario (mediante unos criterios de indicación y admisión, el diagnóstico, evaluación, tratamiento individualizado, finalización y seguimiento); dentro de una garantía de los derechos del usuario; b) integración en un modelo global de prevención y asistencia en drogodependencias (contexto formal y relación con otros servicios); y c) estudio y mejora de la eficacia, eficiencia y efectividad en estos programas (aspectos económicos, evaluación, recursos humanos y garantía de calidad).

En esta misma línea, continúan diversos grupos de trabajo desarrollando proyectos de investigación, que permitan evaluar y redefinir nuevos programas de intervención que se adecuen a las nuevas necesidades de los usuarios. En este momento de inflexión de la Comunidad Terapéutica podemos esbozar algunos indicadores de las nuevas alternativas que comienza a ofrecer este recurso especializado.

\section{Criterios de indicación de comunidad terapéutica.}

Actualmente, la oferta de servicios asistenciales para drogodependientes es relativamente amplia, variada y bien conocida. La elección del contexto y modalidad de intervención debería estar basada en las demandas de un plan de tratamiento, las necesidades del paciente y las características de los servicios disponibles. A priori, los pacientes deberían ser tratados en el entorno menos restrictivo posible respecto al acceso a las sustancias y otras conductas de riesgo, que tenga la mayor probabilidad de ser seguro y efectivo. Las decisiones sobre el lugar de tratamiento deberían tener en cuenta: a) el deseo y capacidad del paciente para cooperar y beneficiarse del tratamiento; b) su necesidad de estructura, apoyo y supervisión para permanecer seguro y alejado de actividades y entornos que le promueven el uso y abuso de sustancias; c) la necesidad específica de tratamientos por comorbilidad médico - psiquiátrica; d) necesidad de tratamientos particulares o una intensidad de tratamiento disponible en entornos específicos; y e) la preferencia por un tratamiento determinado. Los pacientes deberían ser derivados de un nivel asistencial a otro en función de estos criterios y de la evaluación clínica acerca de la disposición del paciente y posibilidad de beneficiarse de un nivel de asistencia de menor intensidad (14). Los estudios que comparan los beneficios relativos de diferentes modalidades de tratamiento presentan diversos problemas metodológicos derivados de la heterogeneidad de las muestras y tipos de programas, altas tasas de abandono, medidas de resultados diferentes, etc. (15). Sin embargo, a continuación se indican algunos criterios que podrían definir la indicación del tratamiento en una comunidad terapéutica para problemas de abuso o dependencia a drogas. Estos criterios de indicación deberían ser objeto de investigación sistemática, con el fin de contribuir a establecer el perfil de usuario más indicado para las CC.TT. y otros programas de asistencia a drogodependientes. 
El rango de servicios disponibles en programas de comunidad terapéutica incluye desintoxicación, evaluación y tratamiento de problemas generales de tipo médico, evaluación psicológica, psicosocial, familiar y vocacional, terapia individual, grupal y familiar, intervención psico- educativa y motivacional, rehabilitación, prevención de recaídas, introducción al concepto y técnicas de autoayuda y elaboración de un plan post tratamiento. La C.T. suele suministrar también (en su entorno o en colaboración con otros recursos) consejo vocacional, preparación para el empleo, tratamiento psicofarmacológico y psiquiátrico. La C.T. ha comenzado ha funcionar como una estructura más flexible, permitiendo el movimiento de pacientes a un entorno abierto, monitorizado aunque menos restrictivo.

Pacientes con comorbilidad psiquiátrica de intensidad moderada; con pobre control de impulsos o con alto riesgo de uso de drogas; con riesgo de sobredosis $u$ otros riesgos para si mismos $u$ otras personas, que no puede ser tratados con seguridad en medio no residencial; pacientes con historia documentada de falta de implicación o beneficio en tratamientos menos intensivos y cuya problemática de abuso constituye riesgo para la salud física o mental; pueden tener indicación de C.T. en régimen más restrictivo, siempre con el consentimiento firmado. Principalmente, el tratamiento en C.T. está indicado para pacientes cuyo funcionamiento vital y e interacción social han venido a enfocarse principalmente o exclusivamente en el uso o abuso de sustancias, o que carecen de la suficiente motivación o apoyo social para mantenerse abstinentes en un entorno ambulatorio, sin reunir criterios para una hospitalización (comorbilidad medica o psiquiátrica severa). Específicamente, los pacientes con dependencia a opiáceos, cocaína, o dependencia de diversas sustancias pueden presentar indicación de tratamiento residencial de duración media o larga, especialmente si hay poca probabilidad de que se beneficien de un tratamiento no residencial $(16,17)$. La C.T. suministra un entorno seguro y libre de drogas de abuso donde el modelamiento conductual y puede ser de especial utilidad para pacientes que necesitan un entorno altamente estructurado para iniciar el tratamiento.

Los criterios de duración del tratamiento residencial deberían ser función del tiempo necesario para alcanzar criterios específicos de aprovechamiento, que a su vez predigan una transición exitosa a un entorno de tratamiento menos restrictivo: por ejemplo, una motivación notoria para seguir un tratamiento ambulatorio, capacidad y motivación para mantener la abstinencia en situaciones de riesgo para la recaída, la disponibilidad de una acomodación y apoyo social que apoye la abstinencia, la estabilización de la comorbilidad médica y psiquiátrica en niveles tratables en régimen ambulatorio, y la existencia de un plan de seguimiento que incluya un plan de readmisión en régimen residencial si fuese necesario.

\section{Adaptación de las CC.TT. a la cronicidad y comor- bilidad de los drogodependientes y a los nuevos patrones de consumo.}

Aunque no existen estudios que evalúen sistemáticamente la comorbilidad psiquiátrica en los usuarios de las CC.TT. Españolas, la evidencia clínica sugiere que un porcentaje cada vez mayor de usuarios presenta trastornos psiquiátricos concurrentes o pasados, especialmente trastornos afectivos y de personalidad. Asimismo, los datos disponibles muestran que los usuarios de C.T. son sujetos que presentan en muchos casos una larga carrera adictiva $y$ antecedentes de múltiples tratamientos seguidos de recaídas (7). Ambas características (cronicidad y comorbilidad) aparecen habitualmente asociadas a peores resultados del tratamiento por abuso de drogas. Consideramos que las CC.TT. profesionalizadas existentes en España podrían beneficiarse de nuevos tratamientos que están demostrando utilidad en pacientes crónicos (por ejemplo, el tratamiento con metadona), y en pacientes con trastornos psiquiátricos concomitantes (medicación psicofarmacológica). Existen algunas experiencias en USA y otros países Europeos que ilustran la adaptación de CC.TT. para pacientes en mantenimiento con metadona y para pacientes con doble diagnóstico, que podrían proporcionar algunas ideas acerca de nuevas modalidades de tratamiento en C.T. La experiencia clínica actual muestra que un porcentaje de pacientes en mantenimiento con metadona no consigue alcanzar los objetivos terapéuticos, y continua con practicas arriesgadas (abuso de cocaína, benzodiacepinas y alcohol, conductas sexuales de riesgo, etc.). Las CC.TT. podrían tener algo que ofrecer para este tipo de pacientes que no se adaptan a programas de tratamiento con metadona en régimen ambulatorio.

Finalmente, aunque las CC.TT. surgieron en América como modalidad de tratamiento principalmente orientadas a los adictos a narcóticos, actualmente la mayoría de nuestros usuarios son politoxicómanos, comenzando a observarse como la heroína, progresivamente deja de ser la droga principal.

\section{VI - CONCLUSIONES}

En nuestro país, los programas basados en el modelo de comunidad terapéutica para drogodependientes están relativamente bien consolidados, los profesionales acumulan años de experiencia con adictos y mantienen una fuerte sensibilidad en la necesidad del cambio y adaptación de los programas de rehabilitación. Están representados principalmente 
por las comunidades terapéuticas profesionales y los programas de la red "Proyecto Hombre".

En la actualidad, existen algunas CC.TT. profesionalizadas que se han ido especializando en tratamientos para los nuevos perfiles de consumidores, desintoxicaciones de metadona, adictos con doble diagnóstico, trastornos de la alimentación, adicciones mixtas (ludopatías - cocaína - alcohol) etc. . Sería deseable que estas experiencias fuesen acompañadas de estudios de evaluación, que puedan ilustrar los beneficios potenciales de las CC.TT., en su adaptación a diferentes poblaciones de consumidores, abusadores y dependientes a diversas sustancias. La aparición de cambios fundamentales en las características de los individuos que presentan trastornos por abuso o dependencia de sustancias (cronicidad, comorbilidad, nuevos patrones de consumo, etc.) y la adaptación a poblaciones específicas (minorías étnicas, mujeres, adolescentes, indigentes, etc.) debe llevar a un cambio adaptativo en las CC.TT. Españolas, al igual que se está produciendo en otros países.

Las perspectivas futuras de las CC.TT. profesionales Españolas se dirigen hacia el debate y la mejora constante de un modelo orientado hacia la reinserción social de los usuarios, no excluyente de otros abordajes, articulados en una red amplia y variada de servicios asistenciales para drogodependientes. Este objetivo se desarrollará a través de una participación activa en la comunicación e intercambio con Intervinientes en las drogodependencias en el ámbito local, regional, nacional e internacional. Los temas de debate serán la calidad en la asistencia a los usuarios; la claridad y estandarización de unas intervenciones basadas en el desarrollo actual del conocimiento racional, técnico y científico; la transparencia en la actuación y la evaluación de nuestra eficacia, eficiencia y efectividad.

El contenido de esta ponencia elaborado para las XXVI Jornadas Nacionales Socidrogalcohol 1999 es un extracto de dos informes recientemente elaborados para su publicación, cuyo contenido más extenso puede ser consultado en:

Llorente, J.M.; Fernández, C. y Gutiérrez M. Las comunidades terapéuticas en el tratamiento del abuso y dependencia de sustancias. Monografías de Psiquiatría. Año 10; Vol.6; pgs.43-53.1998

Fernández, C. y Llorente, J.M. Comunidades terapéuticas para toxicómanos. Nuevas perspectivas. En: Becoña, E.; Rodríguez, A.; Salazar, I.; (Eds.): Drogodependencias, Vol. $n^{\circ} 5$. Universidad de Santiago de Compostela (en prensa). 1999

\section{BIBLIOGRAFIA}

(1) Jones, M.; Therapeutic Community: A New Treatment Method in Psychiatry. New York, Basic Books, 1953.

(2) Polo, L.; Zelaya, M.; Comunidades Terapéuticas para Toxicómanos. Madrid: Dirección General de Acción Social - Cruz Roja, 1985.

(3) Comas, D.; El tratamiento de la drogodependencia y las comunidades terapéuticas. Plan Nacional de Drogas. Ministerio de Sanidad y Consumo. 1987.

(4) Kooyman, M.; The Therapeutic Community for Addicts. Swets \& Zeitlinger. Amsterdam. 1993.

(5) Jones, M.; Más allá de la comunidad terapéutica. Genitor. 1970.

(6) Comas, D; Criterios y normas para la homologación de comunidades terapéuticas profesionales para toxicómanos. APCTT, 1994.

(7) Fernández, C.; Llorente, J.M.; Carrón, J.; Sistema Estándar de Evaluación de la A.P.C.T.T. Asociación de Profesionales de Comunidades Terapéuticas para Toxicómanos. 1995.

(8) Plan Nacional Sobre Drogas. Catálogo de centros públicos o privados con financiación pública de asistencia a drogodependientes. Año 1.997. Madrid. Delegación del Gobierno para el Plan Nacional Sobre Drogas. 1.997.

(9) Llorente, J.M.; Las Comunidades Terapéuticas. CITRAN. Conductas adictivas. $X^{a}$ Reunión adicción a opiáceos. 1997.

(10) Wright, F.D.; Beck, A.T.; Newman, C.F.; Liese, B.S.; Cognitive therapy of substance abuse: theoretical rationale. NIDA Research Monograph 1993; 137:123-146.

(11) Annis, H.M.; Relapse to substance abuse: empirical findings within a cognitive-social learning approach. J. Psychoactive Drugs, 1990 22:117-124.

(12) Stanton, M.D.; Family treatment approaches to drug abuse problems: a review. Fam. Process, 1979; 18:251-280.

(13) Stanton, M.D.; Thomas, T.C.; Family Therapy of Drug Abuse and Addiction. New York, Guilford Press, 1982.

(14) Hoffman, N.G.; Halikas, J.A.; Mee-Lee, D.; Et Al: Patient Placement Criteria for the Treatment of Psychoactive Substance Use Disorders. Washington, DC, American Society of Addiction Medicine, 1991.

(15) Apsler, R.; Harding, W.M. Cost-effectiveness analysis of drug abuse treatment: current status and recommendations for future research. En Background Papers on Drug Abuse Financing and Services Approach: Drug Abuse Services Research Series, number 1, DHHS Publication (ADM) 91-17777. Rockville, Md, National Institute on Drug Abuse, 1991.

(16) Mclellan, A.T.; The psychiatrically severe drug abuse patients: methadone maintenance or therapeutic community? Am. J. Drug Alcohol Abuse, 1984; 10:7795.

(17) De Leon, G.; Rosenthal, M.S.; Treatment in residential therapeutic communities. En Treatments of Psychiatric Disorders: A Task Force. Report of the American Psychiatric Association, vol 2. Washington, DC, APA, 1989. 